\title{
Lapurdum
}

Euskal ikerketen aldizkaria | Revue d'études basques |

Revista de estudios vascos | Basque studies review

Numéro spécial 4 | 2019

SACAZE bildumako euskal testuak (1887)

\section{Herrien zerrenda alfabetikoa}

Gotzon Aurrekoetxea, Charles Videgain et Aitor Iglesias Chaves

\section{OpenEdition \\ Journals}

Édition électronique

URL : https://journals.openedition.org/lapurdum/2942

DOI : 10.4000/lapurdum.2942

ISSN : 1965-0655

Éditeur

IKER

Édition imprimée

Date de publication : 1 octobre 2019

Pagination : 339-345

ISBN : 9782955341339

ISSN : $1273-3830$

\section{Référence électronique}

Gotzon Aurrekoetxea, Charles Videgain eta Aitor Iglesias Chaves, «Herrien zerrenda alfabetikoa», Lapurdum [Linean], Numéro spécial 4 | 2019, Sarean emana----an 12 janvier 2020, kontsultatu 22 septembre 2022. URL: http://journals.openedition.org/lapurdum/2942 ; DOI: https://doi.org/10.4000/ lapurdum.2942

\section{(c) (i) (9)}

Creative Commons - Attribution-NonCommercial-NoDerivatives 4.0 International - CC BY-NC-ND 4.0 https://creativecommons.org/licenses/by-nc-nd/4.0/ 


\section{HERRIEN ZERRENDA ALFABETIKOA}

\subsection{Euskaraz}

Ahatsa / Ahaxe

Ahetze / Ahetze

Aiherra / Ayherre

Ainharbe / Ainharp

Ainhize-Monjolose / Ainhice-Mongelos

Ainhoa / Ainhoa

Aintzila / Aincille

Aiziritze / Aïcirits

Aldude / Les Aldudes

Aloze / Alos-Sibas-Abense

Altzai / Alçay-Alçabéhéty-Sunharette

Altzürükü / Aussurucq

Amenduze / Amendeuix-Oneix

Amorotze-Zokotze / Amorots-Succos

Anhauze / Anhaux

Arberatze-Zilhekoa / Arbérats-Sillègue

Arbona / Arbonne

Arboti-Zohota / Arbouet-Sussaute

Arhantsusi / Arhansus

Armendaritze / Armendarits

Arnegi / Arnéguy

Arrangoitze / Arcangues

Arrueta-Sarrikota / Arraute-Charritte

Arrokiaga / Roquiague 
Arüe / Aroue

Atharratze / Tardets-Sorholus

Atharratze 2 / Tardets-Sorholus 2

Azkaine / Ascain

Azkarate / Ascarat

Baigorri / Saint-Etienne-de-Baïgorry

Banka / Banca

Bardoze / Bardos

Barkoxe / Barcus

Basusarri / Bassussarry

Behaskane-Laphizketa / Béhasque-Lapiste

Behauze / Béguios

Behorlegi / Béhorléguy

Berrogaine-Larüntze / Berrogain-Laruns

Beskoitze / Briscous

Bidarrai / Bidarray

Bidarte / Bidart

Bildoze / Viodos

Biriatu / Biriatou

Bithiriña / Beyrie-sur-Joyeuse

Bunuze / Bunus

Buztintze / Bustince-Iriberry

Domintxaine-Berroeta / Domezain-Berraute

Donaixti-Ibarre / Saint-Just-Ibarre

Donamartiri / Saint-Martin-d'Arberoue

Donapaleu / Saint-Palais

Donazaharre / Saint-Jean-Le-Vieux

Donibane Garazi / Saint-Jean-Pied-de-Port

Donibane Lohizune / Saint-Jean-de-Luz

Donoztiri / Saint-Esteben

Duzunaritze-Sarasketa / Bussunarits-Sarasquette

Eiheralarre / Saint-Michel

Eskiula / Esquiule

Etxarri / Etcharry

Etxebarre / Etchebar

Ezpeleta / Espelette

Ezterenzubi / Estérençuby

Gabadi / Gabat

Gamarte / Gamarthe

Gamere-Zihiga / Camou-Cihigue

Gamue-Zohazti / Camou-Suhast

Garindaine / Garindein

Garrüze / Garris

Getaria / Guéthary

Gotaine-Irabarne / Gotein-Libarrenx 
Haltsu / Halsou

Hauze / Haux

Hazparne / Hasparren

Heleta / Hélette

Hendaia / Hendaye

Hiriburu / Saint-Pierre-d'Irube

Hozta / Hosta

Ibarrola / Ibarrolle

Idauze / Idaux-Mendy

Iholdi / Iholdy

Ilharre / Ilharre

Irisarri / Irissarry

Irulegi / Irouléguy

Iruri / Trois-Villes

Ithorrotze / Ithorots-Olhaïby

Itsasu / Itxassou

Izpura / Ispoure

Izturitze / Isturits

Izura / Ostabat

Jatsu / Jatxou

Jatsu / Jaxu

Jutsi / Juxue

Kanbo / Cambo

Labetze-Bizkai / Labets-Biscay

Lehuntze / Lahonce

Lakarra / Lacarre

Lakarri / Lacarry

Landibarre / Lantabat

Larraine / Larrau

Larresoro / Larressore

Larribarre-Sorhapürü / Larribar-Sorhapuru

Larzabale / Larceveau

Lasa / Lasse

Lekorne / Mendionde

Lekuine / Bonloc

Lekunberri / Lécumberry

Lexantzü-Zunharre / Lichans-Sunhar

Ligi-Atherei / Licq-Athérey

Liginaga / Laguingue-Restoue

Lohitzüne-Oihergi / Lohitzun-Oyhercq

Luhuso / Louhossoa

Lüküze-Altzümarta / Luxe-Sumberraute

Makea / Macaye

Martxueta / Masparraute

Maule / Mauléon 
Mehaine / Méharin

Mendibe / Mendive

Mendikota / Menditte

Milafranga / Villefranque

Mitikile / Moncayolle-Larrory-Mendibieu

Mugerre / Mouguerre

Müsküldi / Musculdy

Oragarre / Orègue

Ortzaize / Ossès

Ospitalepea / L' Hôpital-Saint-Blaise

Ostankoa / Orsanco

Ozaze-Zühare / Ossas-Suhar

Pagola / Pagolle

Santa Grazi / Sainte-Engrâce

Sara / Sare

Sarrikotapea / Charritte-de-Bas

Senpere / Saint-Pée-sur-Nivelle

Sohüta / Chéraute

Suhuskune / Suhescun

Uharte Garazi / Uhart-Cize

Uharte Hiri / Uhart-Mixe

Ündüreine / Espès-Undurein

Urdiñarbe / Ordiarp

Urepel / Urepel

Urketa / Urcuit

Urruña (Pausu) / Urrugne (Béhobie)

Urruña / Urrugne

Ürrüstoi-Larrabile / Arrast-Larrebieu

Uztaritze / Ustaritz

Zalgize-Doneztebe / Sauguis-Saint-Etienne

Zaro / Çaro

Ziburu / Ciboure

Zuraide / Souraïde

\subsection{Frantsesez}

Ahaxe / Ahatsa

Ahetze / Ahetze

Aicirits / Aiziritze

Aincille / Aintzila

Ainharp / Ainharbe

Ainhice-Mongelos / Ainhize-Monjolose

Ainhoa / Ainhoa

Alçay-Alçabéhéty-Sunharette / Altzai

Alos-Sibas-Abense / Aloze 
Amendeuix-Oneix / Amenduze

Amorots-Succos / Amorotze-Zokotze

Anhaux / Anhauze

Arbérats-Sillègue / Arberatze-Zilhekoa

Arbonne / Arbona

Arbouet-Sussaute / Arboti-Zohota

Arcangues / Arrangoitze

Arhansus / Arhantsusi

Armendarits / Armendaritze

Arnéguy / Arnegi

Aroue / Arüe

Arrast-Larrebieu / Ürrüstoi-Larrabile

Arraute-Charritte / Arrueta-Sarrikota

Ascain / Azkaine

Ascarat / Azkarate

Aussurucq / Altzürükü

Ayherre / Aiherra

Banca / Banka

Barcus / Barkoxe

Bardos / Bardoze

Bassussarry / Basusarri

Béguios / Behauze

Béhasque-Lapiste / Behaskane-Laphizketa

Béhorléguy / Behorlegi

Berrogain-Laruns / Berrogaine-Larüntze

Beyrie-sur-Joyeuse / Bithiriña

Bidarray / Bidarrai

Bidart / Bidarte

Biriatou / Biriatu

Bonloc / Lekuine

Briscous / Beskoitze

Bunus / Bunuze

Bustince-Iriberry / Buztintze

Bussunarits-Sarasquette / Duzunaritze-Sarasketa

Cambo / Kanbo

Camou-Cihigue / Gamere-Zihiga

Camou-Suhast / Gamue-Zohazti

Çaro / Zaro

Charritte-de-Bas / Sarrikotapea

Chéraute / Sohüta

Ciboure / Ziburu

Domezain-Berraute / Domintxaine-Berroeta

Etchebar / Etxebarre

Espelette / Ezpeleta

Espès-Undurein / Ezpeize-Ündüreine 
Esquiule / Eskiula

Estérençuby / Ezterenzubi

Etcharry / Etxarri

Gabat / Gabadi

Gamarthe / Gamarte

Garindein / Garindaine

Garris / Garrüze

Gotein-Libarrenx / Gotaine-Irabarne

Guéthary / Getaria

Halsou / Haltsu

Hasparren / Hazparne

Haux / Hauze

Hélette / Heleta

Hendaye / Hendaia

Hosta / Hozta

Ibarrolle / Ibarrola

Idaux-Mendy / Idauze

Iholdy / Iholdi

Ilharre / Ilharre

Irissarry / Irisarri

Irouléguy / Irulegi

Ispoure / Izpura

Isturits / Izturitze

Ithorots-Olhaïby / Ithorrotze

Itxassou / Itsasu

Jatxou / Jatsu

Jaxu / Jatsu

Juxue / Jutsi

L' Hôpital-Saint-Blaise / Ospitalepea

Labets-Biscay / Labetze-Bizkai

Lacarre / Lakarra

Lacarry / Lakarri

Laguingue-Restoue / Liginaga

Lahonce / Lehuntze

Lantabat / Landibarre

Larceveau-Arros-Cibits / Larzabale

Larrau / Larraine

Larressore / Larresoro

Larribar-Sorhapuru / Larribarre-Sorhapürü

Lasse / Lasa

Lécumberry / Lekunberri

Les Aldudes / Aldude

Lichans-Sunhar / Lexantzü-Zunharre

Licq-Athérey / Ligi-Atherei

Lohitzun-Oyhercq / Lohitzüne-Oihergi 
Louhossoa / Luhuso

Luxe-Sumberraute / Lüküze-Altzümarta

Macaye / Makea

Masparraute / Martxueta

Mauléon / Maule

Méharin / Mehaine

Mendionde / Lekorne

Menditte / Mendikota

Mendive / Mendibe

Moncayolle-Larrory-Mendibieu / Mitikile

Mouguerre / Mugerre

Musculdy / Müsküldi

Ordiarp / Urdiñarbe

Orègue / Oragarre

Orsanco / Ostankoa

Ossas-Suhar / Ozaze-Zühara

Ossès / Ortzaize

Ostabat / Izura

Pagolle / Pagola

Roquiague / Arrokiaga

Saint-Martin-d'Arberoue / Donamartiri

Sainte-Engrâce / Santa Grazi

Saint-Esteben / Donoztiri

Saint-Etienne-de-Baïgorry / Baigorri

Saint-Jean-de-Luz / Donibane Lohizune

Saint-Jean-Le-Vieux / Donazaharre

Saint-Jean-Pied-de-Port / Donibane Garazi

Saint-Just-Ibarre / Donaixti-Ibarre

Saint-Michel / Eiheralarre

Saint-Palais / Donapaleu

Saint-Pée-sur-Nivelle / Senpere

Saint-Pierre-d'Irube / Hiriburu

Sare / Sara

Sauguis-Saint-Etienne / Zalgize-Doneztebe

Souraïde / Zuraide

Suhescun / Suhuskune

Tardets-Sorholus / Atharratze

Tardets-Sorholus 2 / Tardets 2

Trois Villes / Iruri

Uhart-Cize / Uharte Garazi

Uhart-Mixe / Uharte Hiri

Urcuit / Urketa

Urepel / Urepel

Urrugne (Béhobie) / Urruña (Pausu)

Ustaritz / Uztaritze 
